\title{
Overall Status of the High Energy Cosmic Radiation Detection Facility Onboard the Future China's Space Station
}

\author{
Yongwei Dong ${ }^{* a \dagger}$, Shuangnan Zhang (PI), Giovanni Ambrosi, for the HERD \\ Collaboration $\ddagger$ \\ E-mail: dongyweihep.ac.cn
}

\begin{abstract}
The High Energy cosmic-Radiation Detection (HERD) facility has been proposed as one of several space astronomy payloads onboard the future China's Space Station, planned for operation starting around 2025 for about 10 years. HERD is a China-led mission with key European contribution led by Italy. The HERD proposal has passed the joint international review organized by the Italian Space Agency and the Center for Space Utilization of the Chinese Academy of Science. The primary scientific objectives of HERD are: Indirect dark matter search with unprecedented sensitivity; precise cosmic ray spectrum and composition measurements up to the knee energy; Gamma-ray monitoring and full sky survey. HERD is composed of 4 scientific instruments. The main one is a homogeneous, almost cubic calorimeter made of about 7500 LYSO cubic crystals and capable of accepting particles incident on its top face and four lateral faces. Each sensitive face is instrumented with a silicon tracker, and covered by a plastic scintillator detector to separate gamma rays from charged particles. Additionally, a transition radiation detector is located on one lateral face for energy calibration of $\mathrm{TeV}$ particles. This design results in an effective geometric factor more than one order of magnitude larger than that of previous missions, and also excellent lepton/hadron separation capabilities thanks to the 3D nature of the calorimeter. The novel design and key specifications of HERD instruments have been successfully verified with beam tests at the CERN SPS.
\end{abstract}

36th International Cosmic Ray Conference -ICRC2019-

July 24th - August 1st, 2019

Madison, WI, U.S.A.

\footnotetext{
${ }^{*}$ Speaker.

$\dagger$ This work was supported by Youth Innovation Promotion Association CAS, No. 2014009; the National Natural Science Foundation of China, Grant No.11327303 and Grant No.11473028; Key Research Program of Frontier Sciences, CAS, Grant NO. QYZDY-SSW-SLH008; the International Partnership Program of Chinese Academy of Sciences, Grant No. 113111KYSB20160053.

$\ddagger$ for collaboration list see PoS(ICRC2019)062
} 


\section{INTRODUCTION}

It is widely accepted that neutral, cold/warm and non-baryonic dark matter (DM) dominates the total matter content in the universe. One way to detect DM is to search in space for its annihilation/decay products, which may lead to characteristic features in the spectra of rare charged $\mathrm{CR}$ components or of gamma-rays. Some circumstantial evidence or hints of anomalies have been reported in recent observations of electron and positron spectra[1, 2, 3, 4, 5]; however, astrophysical sources like pulsars and pulsar wind nebulae can also contribute to these results. New precise measurements of the $e^{+}+e^{-}$energy spectrum and anisotropy in a wider and unexplored energy range will be able to definitively probe the origin of high energy electrons.

The steepening of the primary cosmic ray (CR) spectrum around $\mathrm{PeV}$, the so-called "knee" region, is a classic but still unresolved problem in CR physics since the first observations in 1958[6]. Due to the rapidly falling intensity of the CR flux with energy, experiments with large acceptances and operating over several years are needed to explore the CR spectrum at the PeV energies. Indirect CR measurements, performed by means of ground-based extensive air shower detector arrays, have been therefore used in the past thanks to their large achievable exposures; but they have difficulties in making composition-resolved high-energy resolution measurements of the fine structure of the knee. Conversely, CR measurements in space, which can directly measure the particle charge and energy, have been suffering from small geometrical factors or exposure times preventing them to make statistically meaningful measurements at energies above few TeVs. New space experiments with an exposure of $\sim 20 \mathrm{~m}^{2}$ sryrs are needed to definitively explore the knee structure of the CR spectrum.

Several generations of wide field of view (FOV) space gamma-ray telescopes in the GeV energy regime and ground based narrow FOV gamma-ray telescopes in hundreds of GeV energy regimes have discovered several new populations of astrophysical objects, which allow deeper understanding of the laws of nature under extreme physical conditions only available in cosmic laboratories. In particular the wide FOV space gamma-ray telescopes often provide crucial guidance to the observations of the ground-based narrow FOV telescopes. Unfortunately, the much more powerful ground-based Cherenkov Telescope Array (CTA), currently under development, may not have the needed guidance from a space wide FOV gamma-ray telescope, once the Fermi satellite will stop its operations. A new wide FOV space gamma-ray telescope is urgently needed to replace Fermi.

In order to address the above major problems in fundamental physics and astrophysics, the High Energy cosmic-Radiation Detection (HERD) facility has been proposed as one of several space astronomy payloads onboard the future China's Space Station (CSS), which is planned for operation starting around 2025 for about 10 years.

\section{Sciences and expected performances}

The primary scientific objectives of HERD are: (1) to search for signatures of the annihilation/decay products of dark matter particles in the energy spectra and anisotropy of high energy electrons from $10 \mathrm{GeV}$ to $100 \mathrm{TeV}$ and in the gamma-ray spectrum from $500 \mathrm{MeV}$ to $100 \mathrm{TeV}$; (2) to measure precisely the energy spectra and composition of primary cosmic rays from $30 \mathrm{GeV}$ 
up to PeV in order to determine the mechanism of the knee structure. The secondary scientific objectives of HERD include wide FOV monitoring of the high energy gamma-ray sky from 500 $\mathrm{MeV}$ for gamma-ray bursts, active galactic nuclei and Galactic microquasars.

\subsection{Measurement of cosmic electron spectrum and Dark Matter signal search}

With a larger acceptance, the HERD experiment can measure the electron-positron spectrum with higher precision and at higher energy. As shown in Fig. 1, the nearby astrophysical sources can contribute to the high energy electron-positron spectrum above TeV scale significantly, and induce some obvious spectral features[7]. The simulations show that HERD will be able to detect this spectral feature. The properties of this spectral feature would be important for the CR studies, such as the CR propagation effects near the solar system and the acceleration mechanism at CR sources.
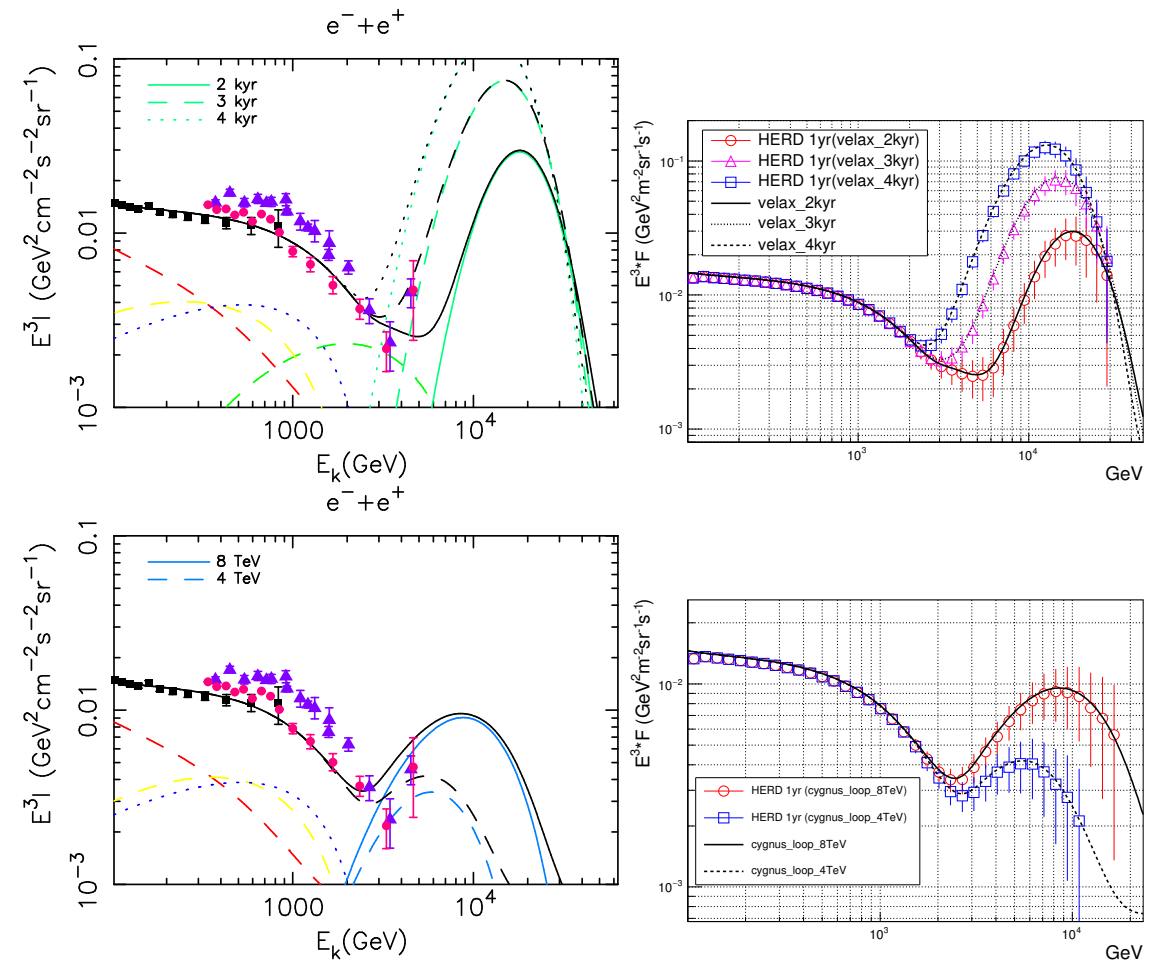

Figure 1: Possible spectrum feature at the $\mathrm{CR}$ electron-positron spectrum above $\mathrm{TeV}$ scale from the nearby sources Vela X (up) and Cgynus loop (down) and the simulated measurement by HERD. The 3 curves for Vela $\mathrm{X}$ are for different electron/positron release time and the 2 curves for Cgynus loop are for different cutoff energy of electron/positron spectrum[7].

For the aim of distinguishing the origins of high energy electrons/positrons from DM and astrophysical sources, the most important advantage of HERD is the anisotropy measurement. In Fig 7, we show the expected anisotropies induced by Vela X and Cygnus Loop. For comparison, the upper-limits provided by Fermi-LAT and the expected sensitivities of HERD are also shown. We can see that the anisotropies induced by these two sources can be detected by HERD, while their predictions are consistent with the AMS-02 spectrum results and the Fermi-LAT anisotropy limits. 
Velax

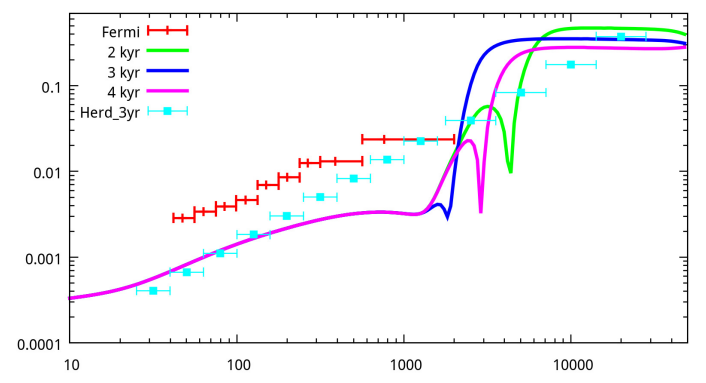

Cygnus_loop

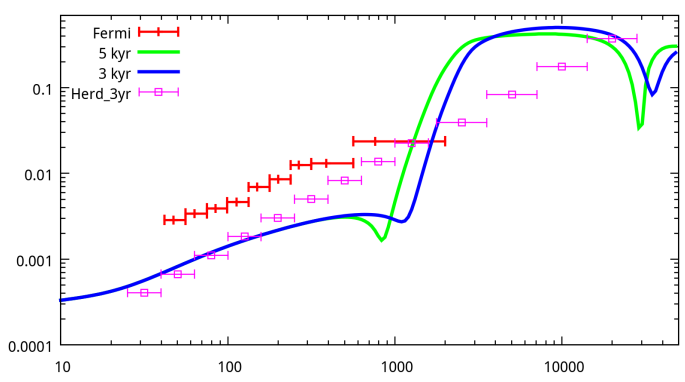

Figure 2: The anisotropy of the CR electron-positron flux considering the contribution from nearby sources Vela X (left) and Cgynus loop (right).

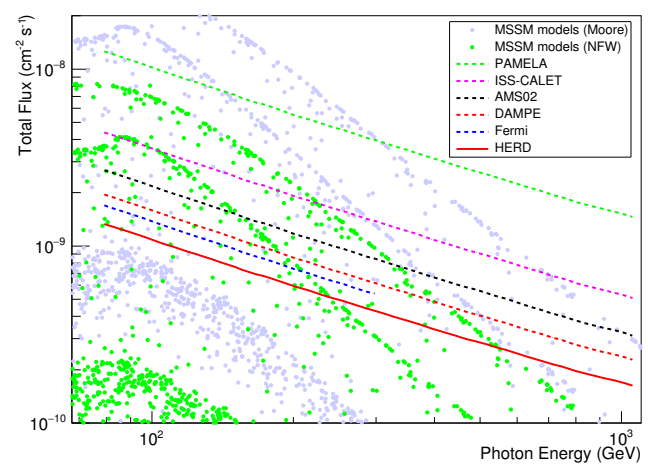

Figure 3: Sensitivity for gamma ray line by different experiments. The background points are theoretical predictions in the MSSM DM model.

The annihilations of DM particles in the galaxy could possibly produce monochromatic gammaray. Such a line shape gamma-ray spectrum is a smoking gun for the detection of DM annihilation. In Fig.3, the sensitivities of gamma-ray line signatures for different experiments are shown. It can be seen that 1-year sensitivity of HERD is better than those of other experiments for more than 5 years. Therefore, HERD has an unprecedented capability for the gamma-ray line signal from DM.

\subsection{Origin of cosmic ray}

It is mandatory to explore the sub-PeV region with high precision direct measurement in order to study the energy spectra of each of the individual nuclear species, to measure the various spectral indices, to detect any possible hardening and to set mass composition below the knee of the allparticle flux. If possible the detection of the steepening of each of the single species and then the explanation of the all-particle knee would be a crucial result in understanding Galactic CR physics, also serving as fundamental input to the study of the extra-galactic component[8].

HERD will be capable of studying features in the spectra of various nuclei, like single element spectral indices and spectral hardenings/steepenings from hundreds $\mathrm{GeV} /$ nucleon up to hundreds $\mathrm{TeV} /$ nucleon. In particular the proton and helium component could be measured up to PeV energies, thus giving the possibility for the first direct evidence of the knee of the light component due 
to the reach of maximum energy provided by the source. This result would be fundamental for understanding Galactic CR acceleration/propagation processes.

Fig. 4 illustrates the HERD measurements of the individual proton and Helium spectrum with a 5-yr exposure, based on a fit to the AMS-02 and ARGO-YBJ data[9]. The HERD data can clearly reveal the knees of both protons and Helium nuclei, and can critically address the $Z$-dependence, $A$-dependence, or constant knee of different compositions, which are very important to understand the physical nature of the knee of CRs.
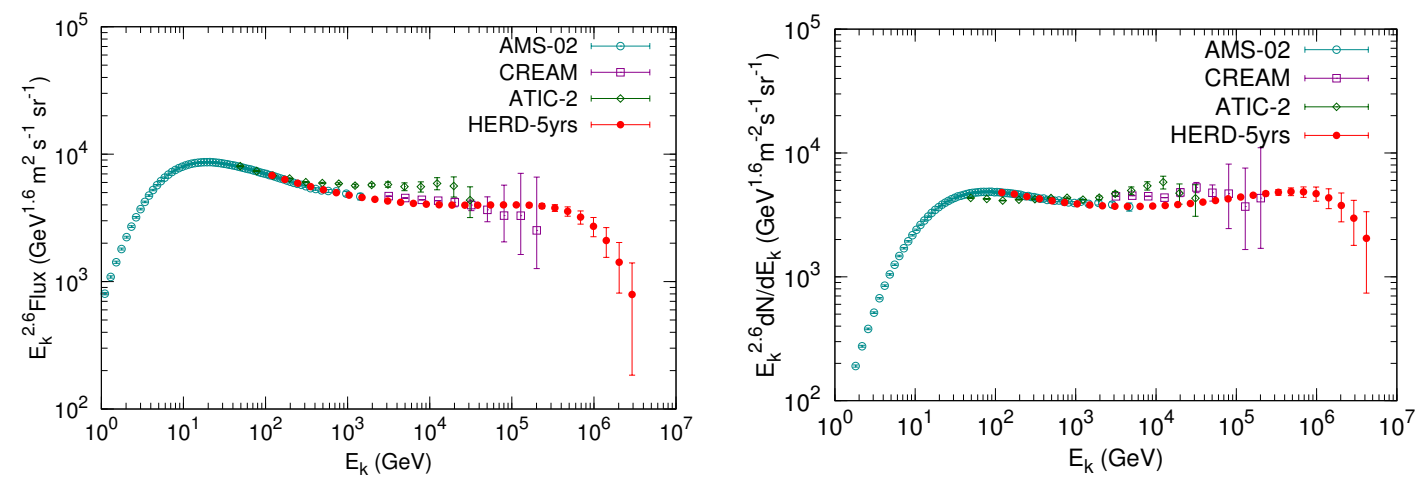

Figure 4: Expected energy spectra of protons (left) and Helium nuclei (right) measured with 5-yr exposure of HERD, compared with other experiments.

HERD will also significantly improve the measurements of the Boron-to-Carbon ratio, Lithium and iron spectra, etc.

\subsection{Gamma ray observatory}

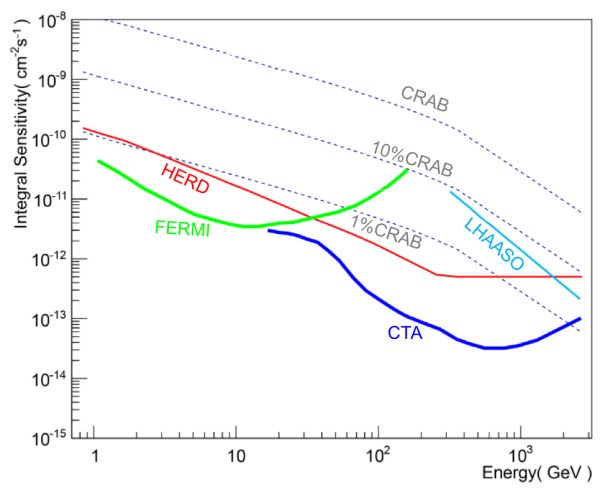

Figure 5: Expected HERD gamma-ray sky survey sensitivity $(5 \sigma)$ in comparison with FERMI LAT, CTA and LHAASO. HERD, FERMI-LAT and LHAASO sensitivities are for one year sky survey. CTA sensitivity is for observations of 50 hours. HERD design with five sides surrounded by seven STK layers is considered. For the baseline design with reduced STK layers for the four lateral sides, the sensitivity is degraded by nearly a factor of 2 .

In Fig. 5 we show the simulated HERD sky survey sensitivity for 1 year continuous operation onboard the CSS, in comparison with the sensitivities of other experiments. It can be seen that 
HERD is the most sensitive sky surveyer with very wide field of view for gamma rays between tens of $\mathrm{GeV}$ and several $\mathrm{TeV}$, thus completely complementing $\mathrm{CTA}$, the most sensitive high energy gamma-ray telescope with a narrow field of view.

The observations in the energy range above several hundreds of MeV by HERD will play a unique and complementary role in multi-wavelength studies across the electromagnetic spectrum with other space and ground telescopes involving radio, optical, X-ray, gamma-ray, and neutrino telescopes.

The multi-messenger astrophysics is born and HERD with its unusual large field of view and unique energy coverage, is well suited to the search of neutrino sources and electromagnetic counterpart of GW.

\section{Mission profile}

The Chinese Space Station is to be completed around 2022 and has a lifetime of more than 10 years. The CSS is normally pointed to the earth under the control of Three-Axis Stabilized system. The CSS, as well as the International Space Station (ISS), is an ideal and powerful platform which supports all-sky survey of high energy cosmic radiation.

Within the framework of Chinese Manned Space Program, HERD will be launched at Wenchang Launching Site by the Long March 5 carrier. After docking onto the CSS, the HERD payload will be transfered by robotic arm to the dedicated mounting point. Payload data are processed by the Information System of Utilization and downloaded through T/C system to ground stations with the help of relay satellites. Some key devices of HERD are planned to be upgraded regularly for the purpose of better performances or to be replaced in case of unexpected failure.

\section{Payload}

In the baseline design[10, 11], HERD is composed of 4 subdetectors (Fig. 6). The main one is a deep 3-D cubic imaging calorimeter (CALO) made of about 7500 cubes of LYSO crystals, which corresponds to from all directions 55 radiation lengths and 3 Nuclear Interaction Lengths, and capable of accepting particles impinging on its top face but also on the four lateral faces.Each sensitive face of the CALO is instrumented with a silicon tracker (STK) to track the direction of the impinging particles and measure their charge. The whole instrument is surrounded by a plastic scintillator detector (PSD), providing gamma-rays and charged particle triggers, as well a redundant charge measurement. A Transition Radiation Detector (TRD), located on one of the lateral sides, is used for the energy calibration of $\mathrm{TeV}$ nuclei.

Thanks to its innovative design, the HERD instrument has a geometrical factor which exceed of more than one order of magnitude what could be achieved in previous missions, while keeping a manageable size/weight of the payload. For the first time, an acceptance $>3 \mathrm{~m}^{2} \mathrm{sr}$ for electrons and gamma-rays and of $>2 \mathrm{~m}^{2} \mathrm{sr}$ for protons and nuclei will be achieved in a space mission, which will insure the collection of a significative statistics up to the highest energies. The wide acceptance will be accompanied by excellent performances in terms of energy resolutions: about $1 \%$ above $200 \mathrm{GeV}$ for $e^{ \pm}$and gamma-ray and of about $20 \%$ for protons and nuclei from $100 \mathrm{GeV}$ to PeV. 


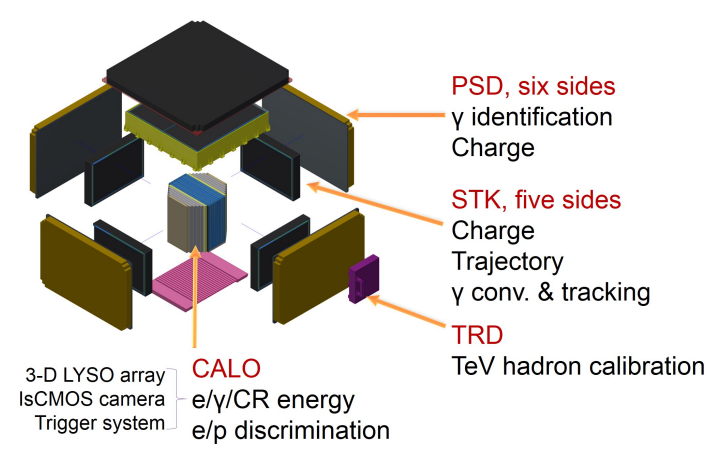

Figure 6: Explosive view of HERD payload

Table 1: HERD main specifications

\begin{tabular}{ll}
\hline \hline Item & Value \\
\hline Energy range (e) & $10 \mathrm{GeV}-100 \mathrm{TeV}$ \\
Energy range $(\gamma)$ & $0.5 \mathrm{GeV}-100 \mathrm{TeV}$ \\
Energy range (nuclei) & $30 \mathrm{GeV}-3 \mathrm{PeV}$ \\
Angular resolution (e/ $\gamma)$ & $0.1 \mathrm{deg} @ 10 \mathrm{GeV}$ \\
Charge measurement (nuclei) & $0.1-0.15 \mathrm{c.u}$. \\
Energy resolution (e) & $1 \% @ 200 \mathrm{GeV}$ \\
Energy resolution (p) & $20 \% @ 100 \mathrm{GeV}-\mathrm{PeV}$ \\
e/p separation & $\sim 10^{-6}$ \\
Geometric factor (e) & $>3 \mathrm{~m}^{2} \mathrm{Sr} @ 200 \mathrm{GeV}$ \\
Geometric factor (p) & $>2 \mathrm{~m}^{2} \mathrm{Sr} @ 100 \mathrm{GeV}$ \\
Lifetime & $>10$ years \\
Pointing & $\mathrm{Zenith}$ \\
Field of View & $+/-70 \mathrm{deg}(\operatorname{targeting}+/-90 \mathrm{deg})$ \\
Envelope & $\sim 2400 * 2400 * 2000 \mathrm{~mm}^{3}$ \\
Weight & $\sim 4000 \mathrm{~kg}$ \\
Power consumption & $\sim 1400 \mathrm{~W}$ \\
\hline \hline
\end{tabular}

With these figures of merit[12], HERD will guarantee direct observations of different CR species and gamma-rays with unprecedented accuracy over a wide energy range.

A novel method of reading out the LYSO signals by Wavelength Shifting Fiber (WLSF) and image intensified CMOS (IsCMOS)[13] is utilized, which can greatly reduce the complexity of onboard electronics. Different technical approaches, as possible alternatives to the current baseline design, are also under study. E.g. dual readout of CALO (WLSF and PD), FIber Track (FIT) and the Tracker In Calorimeter (TIC) techniques.

\subsection{Beam test}

Beam tests of prototypes of HERD detectors were conducted at CERN SPS in 2017 and 2018. A small CALO prototype with 250 LYSO crystals and a big one with 500 crystals were developed 
to verify the performances of electrons and protons. The energy resolution is better than $1.3 \%$ for $200 \mathrm{GeV}$ electrons. And the energy resolution is $21.0 \%$ for $400 \mathrm{GeV}$ protons. The novel design of the 3D calorimeter with WLS fiber + IsCMOS readout is proved to be feasible for broad-band observation with high energy resolution.
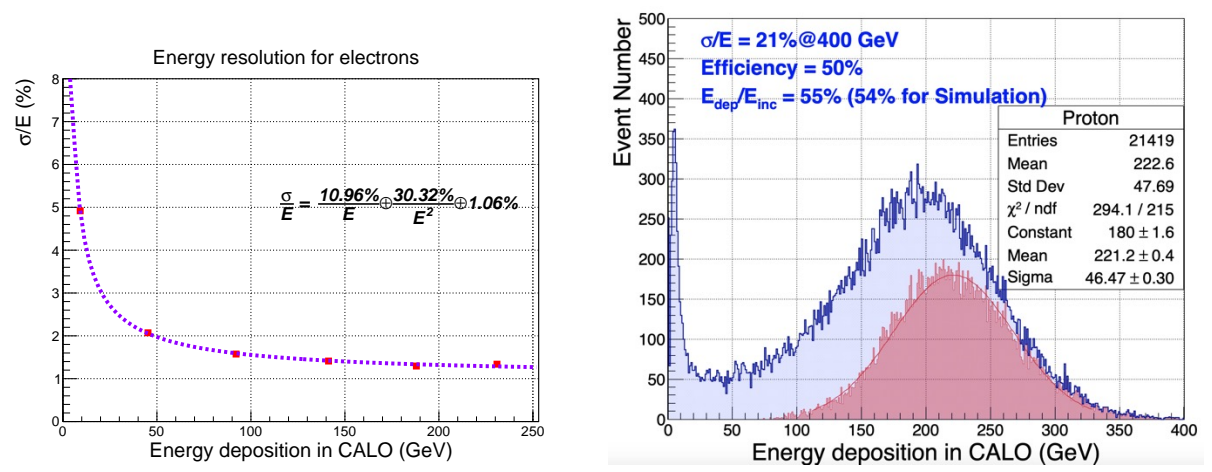

Figure 7: Left: Energy resolution for electrons. The prototype is composed of $5 * 5 * 20$ LYSO crystals. The energy resolution is $1.3 \%$ for $200 \mathrm{GeV}$ electrons. Right: Energy resolution for protons. The prototype is composed of $5 * 5 * 20$ LYSO crystals. With an efficiency of $50 \%$, the energy resolution is $21 \%$ for $400 \mathrm{GeV}$ protons.

During the beam tests, the key functions and performances of other detectors were also well demonstrated.

\section{Summary}

The HERD experiment is focused on the important and frontier sciences in DM search, CR observation and gamma-ray astronomy. The novel design of HERD detectors has been verified in the CERN beam tests. HERD, a China-led mission with a key European contribution led by Italy, will be a flagship and landmark scientific experiment on board the China's Space Station.

\section{References}

[1] M. Aguilar et al., "Precision Measurement of the $\left(e^{+}+e^{-}\right)$Flux in Primary Cosmic Rays from 0.5 $\mathrm{GeV}$ to $1 \mathrm{TeV}$ with the Alpha Magnetic Spectrometer on the International Space Station", Phys. Rev. Lett. 113, 221102 (2014).

[2] O. Adriani et al., "Ten years of PAMELA in space", Riv. Nuovo Cimento 40, 473 (2017).

[3] S. Abdollahi et al., "Cosmic-ray electron-positron spectrum from $7 \mathrm{GeV}$ to $2 \mathrm{TeV}$ with the Fermi Large Area Telescope", Phys. Rev. D 95, 082007 (2017).

[4] G. Ambrosi et al., "Direct detection of a break in the teraelectronvolt cosmic-ray spectrum of electrons and positrons", Nature 552, 63 (2017).

[5] O. Adriani et al., "Extended Measurement of the Cosmic-Ray Electron and Positron Spectrum from $11 \mathrm{GeV}$ to $4.8 \mathrm{TeV}$ with the Calorimetric Electron Telescope on the International Space Station", Phys. Rev. Lett. 120, 261102 (2018).

[6] G. V. Kulikov, G. B. Khristiansen, "On the size spectrum of extensive atmosphere showers", JETP 35, 635 (1958). 
[7] K. Fang et al., "Perspective on the cosmic-ray electron spectrum above TeV", Astrophys. J. 836, 172, (2017).

[8] J. R. Hoerandel, "Models of the knee in the energy spectrum of cosmic rays", Astropart. Phys. 21, 241 (2014).

[9] Y. Q. Guo and Q. Yuan, "On the knee of Galactic cosmic rays in light of sub-TeV spectral hardenings", Chinese Physics C 42.7, 075103 (2017).

[10] S. N. Zhang et al., "The High Energy cosmic-Radiation Detection (HERD) facility onboard China's Space Station", Proc. SPIE, Space Telescopes and Instrumentation 2014: Ultraviolet to Gamma Ray, 9144, 91440X (2014). DOI:10.1117/12.2055280

[11] S. N. Zhang et al., "Introduction to the High Energy cosmic-Radiation Detection (HERD) Facility onboard China's Future Space Station", PoS(ICRC2017)1077. DOI:10.22323/1.301.1077

[12] M. Xu et al., "Monte Carlo Simulation of HERD Calorimeter", Proc. SPIE, Space Telescopes and Instrumentation 2014: Ultraviolet to Gamma Ray, 9144, 91443S (2014). DOI:10.1117/12.2055319

[13] Y. W. Dong et al., "A novel 3-D calorimeter for the High Energy cosmic-Radiation Detection (HERD) Facility onboard China's Future Space Station", PoS(ICRC2017)253. DOI:10.22323/1.301.0253 